\title{
Efecto de Lactobacillus casei microencapsulado sobre la salud intestinal y parámetros bioquímicos y productivos en pollo de engorde
}

\section{Effect of microencapsulated Lactobacillus casei on intestinal health and on biochemical and productive parameters in broiler}

\author{
Henry Jurado-Gámez; ; Edward Johnny Zambrano-Mora²
}

\begin{abstract}
'Zoot. Esp. M.Sc. Ph.D. Universidad de Nariño, Facultad de Ciencias Pecuarias, Departamento de Producción y Procesamiento Animal. Torobajo - Pasto, Colombia; e-mail: henryjugam@gmail.com; Dhttps://orcid.org/0000-0003-2118-7997

${ }^{2}$ M.V. M.Sc. Universidad de Nariño, Facultad de Ciencias Pecuarias, Departamento de Producción y Procesamiento Animal. Torobajo - Pasto, Colombia; e-mail: laboratorio.Idvm@gmail.com; (Dhttps://orcid.org/0000-0002-8443-2243
\end{abstract}

Cómo citar: Jurado-Gámez, H.; Zambrano-Mora, E.J. 2020. Efecto de Lactobacillus casei microencapsulado sobre la salud intestinal y parámetros bioquímicos y productivos en pollo de engorde. Rev. U.D.C.A Act. \& Div. Cient. 23(2):e1480. http://doi.org/10.31910/ rudca.v23.n2.2020.1480

Artículo de acceso abierto publicado por Revista U.D.C.A Actualidad \& Divulgación Científica, bajo una licencia Creative Commons CC BY-NC 4.0

Publicación oficial de la Universidad de Ciencias Aplicadas y Ambientales U.D.C.A, Institución de Educación Superior Acreditada de Alta Calidad por el Ministerio de Educación Nacional.

Recibido: Febrero 1 de 2020 Aceptado: Octubre 1 de 2020 Editado por: Ingeborg Zenner de Polanía

\section{RESUMEN}

El sector avícola ha disminuido los antibióticos por problemas de resistencia, lo que ha generado interés investigativo en alternativas para el manejo sanitario. Por ello, se evaluó el efecto del suministro de Lactobacillus casei microencapsulado en los parámetros productivos y salud intestinal del pollo de engorde. La investigación incluyó 240 pollos, en 4 tratamientos: alimento sin antibióticos ni anticoccidiales (T0), alimento con probiótico comercial (T1) y T2 y T3 con inclusión de $L$. casei microencapsulado y sin microencapsular, respectivamente. Se evaluó la eficiencia y la viabilidad de la conservación de la bacteria por parte del microencapsulado, además de sus características físicas. Se valoró in vivo el suministro de la bacteria láctica, mediante los parámetros productivos, bioquímicos en sangre, la morfología intestinal y la respuesta inmunológica. Se observó viabilidad y eficiencia de 75,9 y 89,4\%, para el microencapsulado.
El grupo de tratamiento con probiótico microencapsulado tuvo incremento significativo en la ganancia de peso y de consumo de alimento $(p<0,05)$. En la bioquímica sanguínea solo se encontró un incremento del colesterol con el microencapsulado $(\mathrm{p}<0,05)$. Se mejoró la respuesta inmunológica con el suministro de $L$. casei microencapsulado $(\mathrm{p}<0,05)$. Se concluye que el uso de $L$. casei microencapsulado puede mejorar los parámetros productivos e inmunológicos del pollo de engorde.

Palabras clave: Microbiología; Probiótico; Pollo de engorde; BAL.

\section{ABSTRACT}

The poultry production has reduced antibiotics due to resistance problems, which has generated research interest in alternatives for sanitary management. Therefore, the effect of supplying 
microencapsulated Lactobacillus casei on the production parameters and intestinal health of broilers was evaluated. The research included 240 chickens in 4 treatments: food without antibiotics or anticoccidials (T0), food with commercial probiotic (T1), and $\mathrm{T} 2$ and $\mathrm{T} 3$ with inclusion of microencapsulated and nonmicroencapsulated $L$. casei, respectively. The efficiency and viability of the conservation of the bacterium by the microencapsulate was evaluated, in addition to its physical characteristics. The supply of lactic acid bacteria was evaluated in vivo by means of the productive, biochemical parameters in blood, intestinal morphology and the immune response. Viability and efficiency of 75.9 and $89.4 \%$ were observed for the microencapsulation. The microencapsulated probiotic treatment group had a significant increase in weight gain and food consumption $(\mathrm{p}<0.05)$. In blood biochemistry, an increase in cholesterol was only found with microencapsulation $(\mathrm{p}<0.05)$. The immune response was improved with the supply of microencapsulated L. casei $(\mathrm{p}<0.05)$. It is concluded that the use of microencapsulated $L$. casei can improve the productive and immunological parameters of broilers.

Keywords: Microbiology; Probiotic; Broiler chickens; LAB.

\section{INTRODUCCIÓN}

El crecimiento de la industria avícola, en las últimas décadas, presenta un crecimiento sostenido del 13\% anual, como resultado de los progresos en mejoramiento genético, nutrición y manejo, que permitió la disminución del tiempo de producción y de los costos; sin embargo, provocó la intensificación de los sistemas avículas (Hamid et al. 2017). Sus efectos ocasionaron desbalances microbianos, a nivel entérico, que repercute sobre la productividad de las aves (Milian, 2008). Por ello, la industria avícola implementa planes profilácticos con antibióticos en las dietas de los animales, para disminuir los problemas sanitarios y favorecer el aprovechamiento de nutrientes (Gunnarsson \& Mie, 2018).

El uso indiscriminado de antibióticos incrementó la resistencia de muchas cepas patógenas a estos medicamentos, siendo un problema de salud pública, a nivel mundial. En respuesta a estas evidencias, muchos países prohibieron su utilización, como aditivo en las dietas de animales de granja, lo que impulsó la búsqueda de nuevas alternativas. Investigaciones documentan que los probióticos son una opción sustituta de antibióticos en este campo y el mejoramiento de la salud intestinal de los animales (Al-Khalaifah, 2018).

Dentro de los probióticos, las bacterias ácido lácticas (BAL), se han considerado como alternativa a los antibióticos (Ghasemian et al. 2018). Eckert et al. (2017) mencionan que, para optimizar la viabilidad de las BAL en el tracto digestivo, la cepa debe ser microencapsulada, mejorando su tránsito por el tracto digestivo, además de facilitar los procesos de industrialización, como aumento del tiempo de almacenamiento y su liberación in situ. Esta investigación determinó el efecto del suministro de $L$. casei microencapsulado sobre la salud intestinal y los parámetros bioquímicos y productivos en pollos ROSS 308 AP.

\section{MATERIALES Y METODOS}

El estudio fue realizado en las instalaciones de la Universidad de Nariño, en el laboratorio de investigación FISE-PROBIOTEC, que se encuentra a los $2.560 \mathrm{~m}$ s.n.m., con temperatura promedio de $15^{\circ} \mathrm{C}$ y humedad relativa del 75\% (IDEAM, 2019).

Se empleó la cepa L. casei ATCC 8014, que se reconstituyó en agar MRS, de acuerdo con la casa comercial; luego de $24 \mathrm{~h}$ de reconstituida, se confirmó su crecimiento y desarrollo, y se repicó con asa de argolla, en cajas de agar MRS comercial, con azul de anilina y se incubó por $24 \mathrm{~h}$, a $37^{\circ} \mathrm{C}$. De manera paralela, se hizo repique en tubos con caldo MRS, para lo cual, se tomó una alícuota del reconstituido y se adicionó a tubos de ensayo, con $10 \mathrm{~mL}$ de caldo MRS, que enseguida fueron llevados a incubación en el tiempo y temperatura de $32^{\circ} \mathrm{C}$, propuestos para el método de siembra en caja. $\mathrm{Al}$ terminar la incubación, se tomaron muestras de cada caja y tubo de ensayo y se evaluó la morfología macroscópica y microscópica de la BAL, mediante coloración de Gram (Rodríguez \& Arenas, 2018).

Para obtener el inóculo de L. casei, se utilizaron los parámetros de cinética de crecimiento y el procedimiento, descritos en JuradoGámez et al. (2014), quienes determinaron la fase exponencial a las 16h, con un crecimiento de $7,3 \times 10^{8} \mathrm{UFC} / 150 \mathrm{~mL}$, en medio MRS.

Se determinó el crecimiento de L. casei bajo las temperaturas de 37 y $45^{\circ} \mathrm{C}$ (Cai et al. 1999) y se evaluaron la producción de gas y la actividad de catalasa de la cepa (Cai et al. 1999; Dahl et al. 1989).

Para microencapsular la bacteria, primero, se obtuvo la biomasa microbiana, mediante incubación, a $37^{\circ} \mathrm{C}$ en caldo MRS, por $24 \mathrm{~h}$ (condiciones aerobias) y, al final de la fase exponencial (16h), se centrifugó el incubado, a $5.000 \mathrm{rpm}\left(4^{\circ} \mathrm{C}\right)$ y se descartó el sobrenadante. Después, la muestra fue lavada con una solución estéril de $\mathrm{NaCl}$ al $0,85 \% \mathrm{p} / \mathrm{v}$ y conservada en refrigeración $\left(4 \pm 2^{\circ} \mathrm{C}\right)$, hasta posterior uso.

El proceso de encapsulación, se realizó de acuerdo con la metodología de Rodríguez-Barona et al. (2016). Para ello, se preparó un inóculo con $400 \mathrm{~mL}$ al $15 \% \mathrm{p} / \mathrm{v}$ de $\mathrm{L}$. casei $(60 \mathrm{~g}$ de maltodextrina y $60 \mathrm{~g}$ de Inulina en $28 \mathrm{~mL}$ de inóculo bacteriano, previamente ajustado), en relación 1:1 p/p, que fue agitado hasta homogenizar. Enseguida, la muestra se llevó a un equipo de secado por aspersión (Secador Spray Bilon $6000 \mathrm{~s}^{\mathbb{B}}$ ), con una temperatura de entrada de $170^{\circ} \mathrm{C}$ y una temperatura de salida entre 65 y $67^{\circ} \mathrm{C}$, con ciclo completo de $2 \mathrm{~h}$ y $30 \mathrm{~min}$. El material microencapsulado, se empacó en recipientes plásticos oscuros estériles y se almacenó a temperatura ambiente $\left(20 \pm 2^{\circ} \mathrm{C}\right)$.

Se determinó la viabilidad y la sobrevivencia (eficiencia) de la cepa, luego de microencapsulada (Rodríguez-Barona et al. 2016); de igual manera, las características físicas: humedad, actividad de agua, solubilidad y humectabilidad (Rodríguez-Barona et al. 2016). Se estableció la morfología y el tamaño del microencapsulado, mediante microfotografía electrónica. 
Se evaluó el crecimiento de la cepa microencapsulada a concentraciones de 0,1 y $0,3 \%$, de bilis y 0,5 y $1 \%$, de sales biliares, en condiciones in vitro (Coppola \& Gil-Turnes, 2004).

Una vez establecida la carga microbiana fue adicionarla por aspersión a una concentración de $20 \%$ p/v. Finalmente, el alimento se incubó a $37^{\circ} \mathrm{C}$, por $24 \mathrm{~h}$ y se conservó a temperatura ambiente.

La cepa microencapsulada fue evaluada in vivo. Se usaron 240 pollos machos de un día de edad (Ross 308 AP), que se alojaron en jaulas metálicas. Los pollos, se recibieron a una temperatura de $32^{\circ} \mathrm{C}$, que disminuyó de forma paulatina a $21^{\circ} \mathrm{C}$, durante los primeros 15 días de evaluación. La humedad, se mantuvo entre el 50 y 65\%. Durante los primeros 7 días, los pollos tuvieron luz las 24h, para luego ir disminuyendo gradualmente hasta $12 \mathrm{~h}$, al tercer día. El experimento, se realizó de los 7 a los 35 días y el agua y el alimento se suministraron ad libitum.

Las aves, se distribuyeron de manera aleatoria en cuatro grupos de 60 aves, cada uno. El primer grupo fue alimentado con una dieta comercial sin aditivos (T0); el segundo, dieta comercial con probiótico comercial (T1); el tercero, dieta comercial con L. casei microencapsulado (T2) y el cuarto, dieta comercial con L. casei $\sin$ microencapsular (T3).

Para la evaluación in vivo, se determinó consumo de materia seca (CMS), ganancia de peso (GP) y conversión alimenticia (CA) de las aves. $\mathrm{Al}$ igual que las variables de bioquímica sanguínea: triglicéridos, colesterol y proteínas totales.

Al finalizar el estudio, se sacrificaron 6 aves por grupo y se tomaron muestras de duodeno, yeyuno e íleon, las cuales, se colocaron en formol buferado al 10\%, para fijación por $24 \mathrm{~h}$. Con las muestras, se determinó histopatología, microscopia electrónica y tinciones especiales (Alcian blue, Gram, y hematoxilina y eosina), con el fin de determinar problemas, a nivel morfológico. La tinción de Alcian blue, se ajustó a un pH de 3,0 y se realizó conteo de células caliciformes, observando 10 campos por muestra. Los tejidos, se observaron en objetivo 40x con microscopio Nikon Eclipse 80i, camera infinity y el software ImagePro Plus 5.0 software (Media Cybernetics, Silver Spring, MD, USA).

Las muestras del segmento conservado en alcohol al $90^{\circ} \mathrm{C}$ fueron enviadas al Centro de Microscopia y Microanálisis de la Universidad Nacional, en Bogotá, Colombia, para realizar el registro fotográfico.

Las variables fueron caracterizadas mediante estadística descriptiva, mientras que los parámetros productivos (GP, CA y CMS), bioquímica sanguínea (triglicéridos, colesterol y proteínas totales) y el crecimiento a diferentes concentraciones de sales biliares y bilis, se evaluaron con un diseño completamente al azar (DCA) y las diferencias fueron determinadas mediante la prueba de Tukey, a un nivel de significancia del 95\%.

\section{RESULTADOS Y DISCUSIÓN}

El crecimiento de la bacteria fue de $3 \times 10^{12}$ y $4 \times 10^{12} \mathrm{UFC} / \mathrm{mL}$, a 37 y $45^{\circ} \mathrm{C}$, respectivamente. Matos et al. (2017) y Palachum et al. (2018) encontraron crecimientos superiores a $3 \times 10^{10}$ y 2 × $10^{12}$ $\mathrm{UFC} / \mathrm{mL}$, a temperaturas de 38 y $42^{\circ} \mathrm{C}$, respectivamente, valores menores al obtenido en el presente estudio. Las aves tienen una temperatura interna promedio de $41^{\circ} \mathrm{C}$, por lo que los resultados a $45^{\circ} \mathrm{C}$ indican viabilidad de $L$. casei a las condiciones internas de las aves (Matos et al. 2017).

Los resultados de la microencapsulación, se observan en la tabla 1. La viabilidad durante un periodo de 20 días fue buena $(75,94 \%)$, lo que señala que la técnica de microencapsulación fue efectiva y podría ser una alternativa para su uso en campo. El estudio de Wang et al. (2018) encontró una viabilidad de 67\% a los 15 días de la microencapsulada, que se administró por aspersión, lo que permite inferir un mayor tiempo de conservación de la cepa.

En cuanto a la eficiencia al momento de recuperar la cepa microencapsulada, muestra un valor de 89,36\%. Parra-Huertas (2010) menciona que los resultados de la técnica deben estar entre 96 a 100\%, lo que muestra un menor porcentaje de eficiencia en el microencapsulado obtenido (Tabla 1); sin embargo, el mismo

Tabla 1. Variables de viabilidad, eficiencia y propiedades física del microencapsulado de L. casei mediante la técnica de Spray Drive.

\begin{tabular}{|l|c|c|}
\hline \multicolumn{1}{|c|}{ Factor } & Valor obtenido & Tiempo (d) \\
\hline Viabilidad & $75,94 \%$ & 20 \\
\hline Eficiencia & $89,36 \%$ & 20 \\
\hline \multirow{2}{*}{ Humedad relativa } & $13,85 \%$ & 22 \\
\cline { 2 - 3 } & $5,23 \%$ & 35 \\
\hline \multirow{2}{*}{ Actividad de agua } & 0,353 & 22 \\
\hline \multirow{2}{*}{ Humectabilidad } & 0,407 & 35 \\
\hline Solubilidad & $2: 24 \mathrm{~min}$ & 31 \\
\cline { 2 - 3 } & $3: 08 \mathrm{~min}$ & 12 \\
\hline
\end{tabular}


autor menciona que la estructura de la pared y las condiciones de producción del microencapsulado (temperatura, $\mathrm{pH}$, presión, humedad) tienen un efecto significativo sobre la eficiencia. Por otra parte, Gómez-Mascaraque et al. (2017) mostró una eficiencia del $87 \%$, utilizando la misma técnica, lo que podría indicar que los valores están dentro de lo esperado.

En cuanto a las características físicas (Tabla 1), se encontró una menor humedad de 5,23, a los 35 días de evaluación. Este resultado es importante, dado que el parámetro influye sobre la conservación de la cepa microencapsulada y el tiempo en que es viable, para ser administrada (Champagne et al. 2017). En cuanto a la actividad del agua (Aw), los resultados están dentro de lo establecido por Muhammad et al. (2017), quien menciona que este parámetro debe estar alrededor de 0,3, para evitar el deterioro de la cápsula, por parte de microorganismos del ambiente. La humectabilidad está por encima de 2 minutos, que es el valor que reporta Champagne et al. (2017), para una adecuada humectación y consiguiente liberación de la bacteria, ya que permite mejorar la sobrevivencia. La solubilidad mostró excelentes resultados, dado que este parámetro mide la capacidad de disolverse en el agua, factor importante para una correcta liberación del microorganismo en el tracto gastrointestinal.

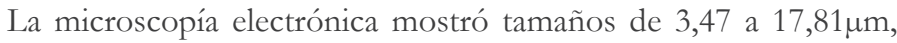
para el microencapsulado (Figura 1), valor cercano al observado por Wang et al. (2018), con 7 a $15 \mu \mathrm{m}$, en L. delbruekii sub. bulgaricus; por

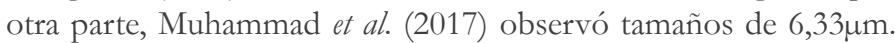
Lo anterior demuestra que el tamaño se encuentra cercano a lo reportado por la literatura.

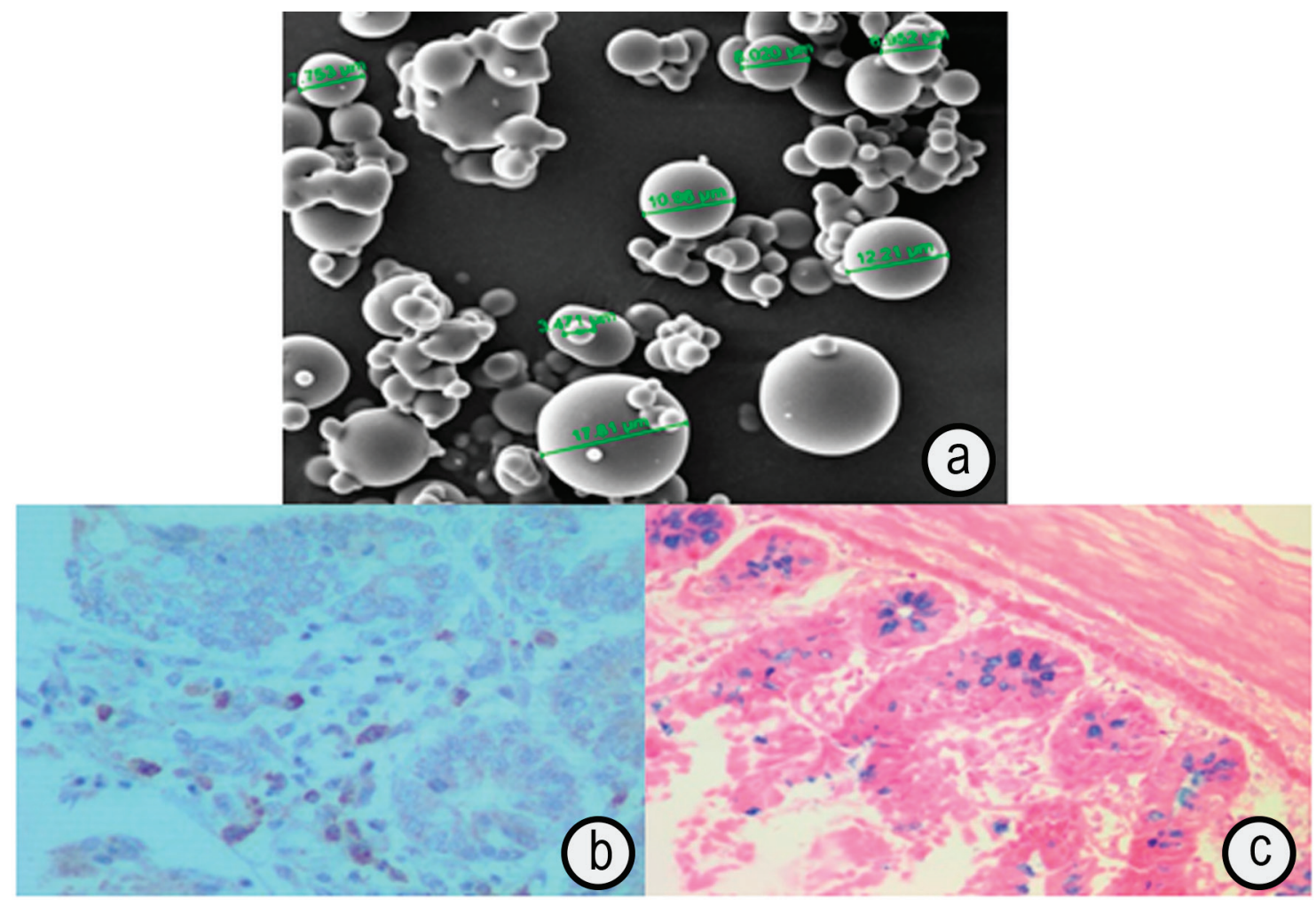

Figura 1. Microfotografía de barrido de L. casei microencapsulado por la técnica de Spray Drying y tinción Alcian blue para caliciformes. a. tamaño del microencapsulado; b. lamina de inmunohistoquímica y c. tinción de Alcian blue para caliciformes.

Los resultados de bilis y sales biliares en condiciones in vitro, se observan en la figura 2. Se encontró una disminución en el crecimiento de la cepa por efecto de las concentraciones de bilis y sales biliares, lo que evidencia dificultades para adaptarse a estas condiciones. Overbeck et al. (2017) manifiestan que la resistencia a $\mathrm{pH}$ básico permite a la bacteria atravesar el intestino delgado, lugar donde se libera la bacteria microencapsulada.

Los parámetros productivos, se pueden observar en la tabla 2 . Los resultados indican que los animales con suministro de $L$. casei microencapsulado mostraron mejores resultados para GP y CMS $(p<0,05)$, aunque no hubo diferencia para la CA $(p>0,05)$. Esto es importante para los sistemas de producción, dado que un mayor consumo trae, como consecuencia, una mejor ganancia de peso y mejora la rentabilidad del sistema (Jones et al. 2019). Al respecto, Stefaniak et al. (2019) encontraron un efecto positivo del suministro de L. plantarum sobre los parámetros productivos de los pollos de engorde, lo que corrobora los resultados obtenidos y permite evidenciar la importancia de las BAL en los sistemas de producción avícola. 


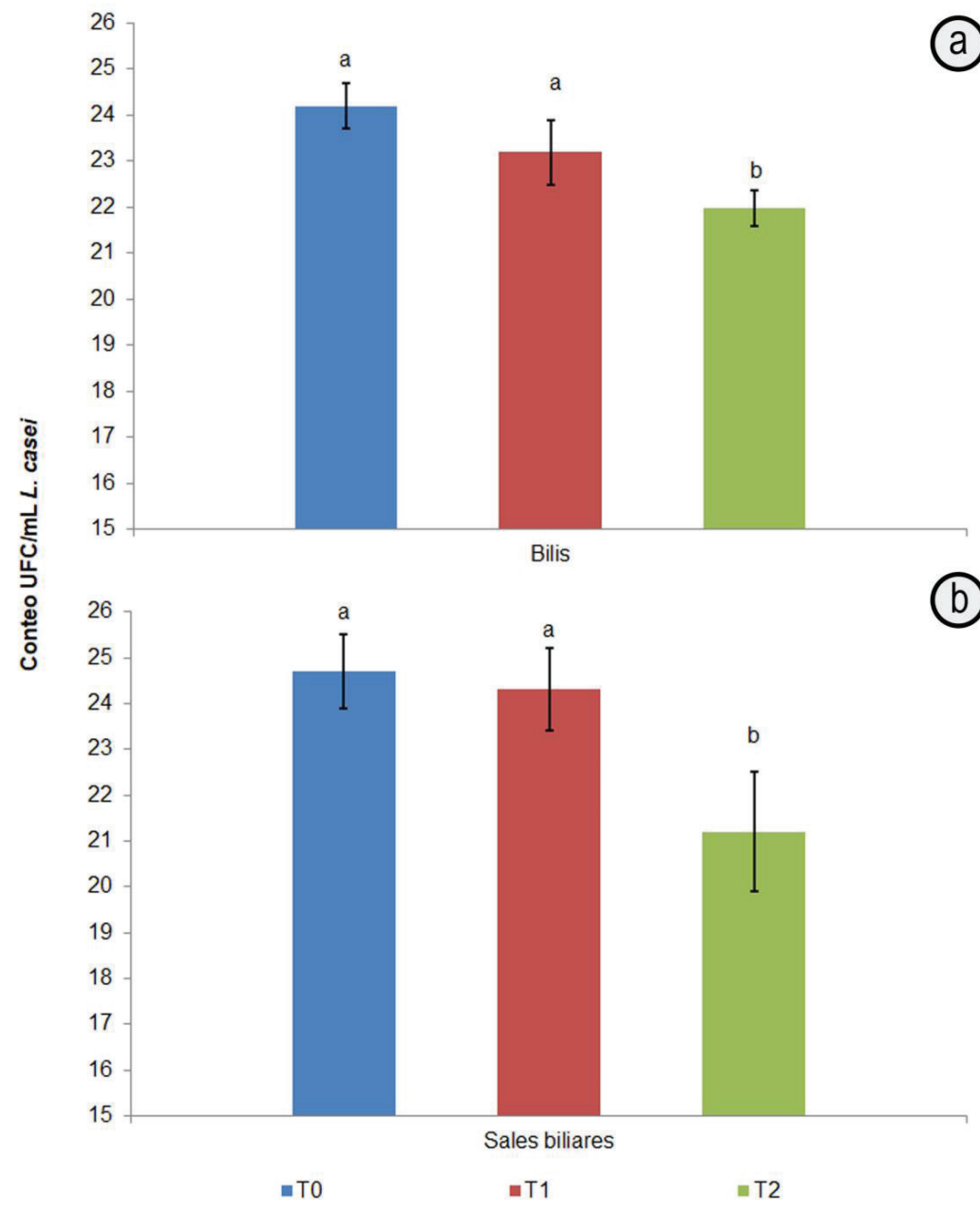

Figura 2. Evaluación del crecimiento in vitro de L. casei, a concentraciones de: a. 0,5, 1 y 1,5\% de bilis y b. 0,3, 0,5 y 1,0 de sales biliares.

Tabla 2. Resultados obtenidos para los parámetros productivos, bioquímicos, microbiológicos e histológicos en pollos de engorde, alimentados con L. casei.

\begin{tabular}{|l|c|c|c|c|}
\hline \multicolumn{1}{|c|}{ Variable } & T0 & T1 & T2 & T3 \\
\hline GP $(\mathrm{g})$ & $181,2 \pm 31,04^{\mathrm{b}}$ & $240,4 \pm 17,94^{\mathrm{ab}}$ & $282,4 \pm 66,03^{\mathrm{a}}$ & $175,0 \pm 24,3^{\mathrm{b}}$ \\
\hline Consumo $(\mathrm{g})$ & $4403,0 \pm 407,7^{\mathrm{b}}$ & $4313,3 \pm 283,34^{\mathrm{b}}$ & $5630,0 \pm 289,32^{\mathrm{a}}$ & $3836,3 \pm 301,21^{\mathrm{b}}$ \\
\hline $\mathrm{CA}$ & $4,39 \pm 0,07^{\mathrm{a}}$ & $3,21 \pm 0,17^{\mathrm{a}}$ & $3,54 \pm 0,27^{\mathrm{a}}$ & $4,24 \pm 0,34^{\mathrm{a}}$ \\
\hline Colesterol $\mathrm{mg} / \mathrm{dl}$ & $158,8 \pm 0,34^{\mathrm{b}}$ & $161,5 \pm 0,56^{\mathrm{b}}$ & $170,0 \pm 0,39^{\mathrm{b}}$ & $191,6 \pm 0,35^{\mathrm{a}}$ \\
\hline Triglicéridos $\mathrm{mg} / \mathrm{dl}$ & $116,0 \pm 0,45^{\mathrm{a}}$ & $115,5 \pm 0,51^{\mathrm{a}}$ & $92,5 \pm 0,31^{\mathrm{a}}$ & $91,8 \pm 0,42^{\mathrm{a}}$ \\
\hline Proteína total $\mathrm{g} / \mathrm{dl}$ & $3,48 \pm 0,03^{\mathrm{a}}$ & $3,77 \pm 0,04^{\mathrm{a}}$ & $3,57 \pm 0,06^{\mathrm{a}}$ & $4,13 \pm 0,08^{\mathrm{a}}$ \\
\hline L, casei $(\mathrm{UFC} / \mathrm{mL})$ & $9,5 \times 10^{5 \mathrm{~b}}$ & $2,84 \times 10^{7 \mathrm{a}}$ & $4,87 \times 10^{\mathrm{a}}$ & $3,36 \times 10^{7 \mathrm{a}}$ \\
\hline Coliformes totales (UFC/mL) & $6,67 \times 10^{2 \mathrm{~b}}$ & $5,57 \times 10^{4 \mathrm{a}}$ & $4,5 \times 10^{3 \mathrm{~b}}$ & $2,83 \times 10^{3 \mathrm{~b}}$ \\
\hline E, coli (UFC/mL) & 0 & 0 & 0 & 0 \\
\hline Inmunohistoquímica* & $367 \pm 55^{\mathrm{a}}$ & $226 \pm 53^{\mathrm{b}}$ & $27 \pm 9^{\mathrm{c}}$ & $143,5 \pm 46^{\mathrm{b}}$ \\
\hline Caliciformes** & $1476 \pm 145^{\mathrm{a}}$ & $957 \pm 137^{\mathrm{a}}$ & $1434 \pm 189^{\mathrm{a}}$ & $1385 \pm 199^{\mathrm{a}}$ \\
\hline
\end{tabular}

T0: testigo; T1: probiótico comercial; T2: L, casei microencapsulado; T3: L, casei sin microencapsular;

* recuento de linfocitos B en intestino delgado (10 campos);

**recuento de células caliciformes en 10 campos. Media \pm desviación estándar.

Letras diferentes en filas muestran diferencias estadísticas $(\mathrm{p}<0,05)$. 
Los resultados de los parámetros bioquímicos, se pueden ver en la tabla 2 (colesterol, triglicéridos y proteína total). Se observó diferencia estadística en los niveles de colesterol en sangre $(p<0,05)$, con un valor más alto en el tratamiento con $L$. casei sin microencapsular (T3), mientras que los niveles de triglicéridos y proteína total fueron similares ( $\mathrm{p}>0,05)$. Al respecto, el estudio de Wang et al. (2019) halló una reducción en los niveles de colesterol en sangre, por efecto del suministro de cepas de lactobacilos en la dieta de ratones. Por otra parte, Abbas et al. (2018) encontraron que la adición de probióticos no tuvo efecto sobre los niveles de colesterol, pero fue positivo sobre el contenido de proteínas totales. Estos resultados demuestran que el efecto de los probióticos sobre la bioquímica sanguínea aún no se comprende del todo y que los resultados son disímiles para estos parámetros; sin embargo, L. casei solo tuvo efecto sobre el colesterol, incrementando su valor sanguíneo.

Los resultados microbiológicos, se observan en la tabla 2 ( $L$. casei, coliformes totales y E. coli). Se evidenció mayor presencia de Lactobacillus en los tratamientos suplementados con probióticos en comparación con el testigo $(\mathrm{p}<0,05)$. Los coliformes totales muestran una población representativa mayor para el T1 $(p<0,05)$, mientras que no hubo presencia de E. coli en las muestras. Según Sobczak et al. (2018), las poblaciones innatas de BAL decrecen desde la semana $1\left(6,5 \times 10^{6}\right)$ hasta la semana $6\left(2,3 \times 10^{4}\right)$ y, en este estudio, se encontró que $L$. casei se instaló con poblaciones de 1,37 x $10^{7}$ (alimento con microencapsulado) y $2,36 \times 10^{7}$ (alimento con $L$ casei sin microencapsulado), por lo que se verifica su colonización. Lo anterior muestra un impacto únicamente en el incremento de BAL en el tracto gastrointestinal de las aves.

Los títulos de New Castle a los 35 días de evaluación fueron mayores para el tratamiento $\mathrm{T} 3$, seguido del tratamiento $\mathrm{T} 1(\mathrm{p}<0,05)$, mientras que Gumboro tuvo niveles altos para los tratamientos T0 y T3, siendo inferiores los tratamientos T1 y T2 (a 35 días, $\mathrm{p}<0,05$ ). Para los títulos de bronquitis, se observaron mayores valores para los tratamientos T2 y T3 (a 35 días, p<0,05) (Figura 3). Se ha encontrado que $L$. casei mejora la respuesta inmunitaria, reduce el riesgo de infección en el tracto respiratorio, estimula las células NK del huésped y compite con microorganismos patógenos, lo que corrobora los resultados observados (Zhang et al. 2016). Además, los resultados fueron tomados al final de la evaluación, por lo que los efectos se mantienen durante toda la evaluación.

Los resultados de la histopatología, se observan en la figura 4. Se encontraron mayores alteraciones celulares en los tratamientos con suministro de L. casei, como hiperplasia de epitelio, que estuvo presente en el 100\% de las muestras del yeyuno, de los tratamientos
T2 y T3; hiperplasia de glándulas, que se presentó en los tratamientos T2 y T3, en un 50\% e hiperplasia de caliciformes, con 50\% de las muestras, en los mismos tratamientos; al parecer, la administración del probiótico tuvo un efecto sobre la presentación de estas lesiones. Según Castro-González et al. (2019), la bacteria láctica influye no directamente sobre la aparición de las lesiones, sino que estas bacterias, en casos poco frecuentes, incrementan estas lesiones, como consecuencia de un elevado crecimiento poblacional, lo que demuestra un exceso de bacteria en el inóculo, por lo que se debe evaluar este parámetro.

En los recuentos de linfocitos B (inmunohistoquímica), que están expresando proteínas para $\operatorname{IgA}$, se encontró que el tratamiento T0 (testigo) tiene mayor cantidad de estas células (Figura 1 y Tabla 2), mientras que, por el contrario, el tratamiento que recibió el microencapsulado (T3), expresó la menor cantidad. Este hallazgo es muy importante, ya que demuestra que $L$ casei realiza inmunomodulación en el tejido intestinal de aves, contrario a lo reportado por la mayoría de autores (Castro-González et al. 2019), quienes atribuyen a los BAL un aumento de la inmunidad humoral local en intestino delgado.

La marcación de células caliciformes con Alcian Blue fue efectiva en los pollos (Figura 1), lo que demuestra que la técnica también puede ser utilizada en este tipo de tejido. En la tabla 2, se observa que hay un menor conteo en el T1. Al respecto, Bingle et al. (2010) mencionan que las células caliciformes se encargan de secretar moco y se encuentran, tanto en las vellosidades como en las criptas. Su importancia radica en que presentan variaciones, de acuerdo con factores ambientales y clínicos, lo que permite identificar determinadas patologías. El conteo de caliciformes no mostró diferencias estadísticas ( $\mathrm{p}>0,05)$, lo que evidencia un comportamiento similar de los tratamientos en este parámetro.

Finalmente, la microencapsulación de $L$. casei incrementa la ganancia de peso y mejora la respuesta inmunológica; sin embargo, se encontraron lesiones con el suministro de la bacteria, lo que indicó que se debe ajustar la dosis, la frecuencia y la vía de suministro, para evitar este tipo de alteraciones.

Conflicto de Intereses: El manuscrito fue preparado y revisado con la participación de todos los autores, quienes declaramos que no existe ningún conflicto de intereses que ponga en riesgo la validez de los resultados presentado. Financiación: Este estudio fue financiado por la Vicerrectoría de Postgrados y Relaciones Internacionales VIPRI de la Universidad de Nariño. 


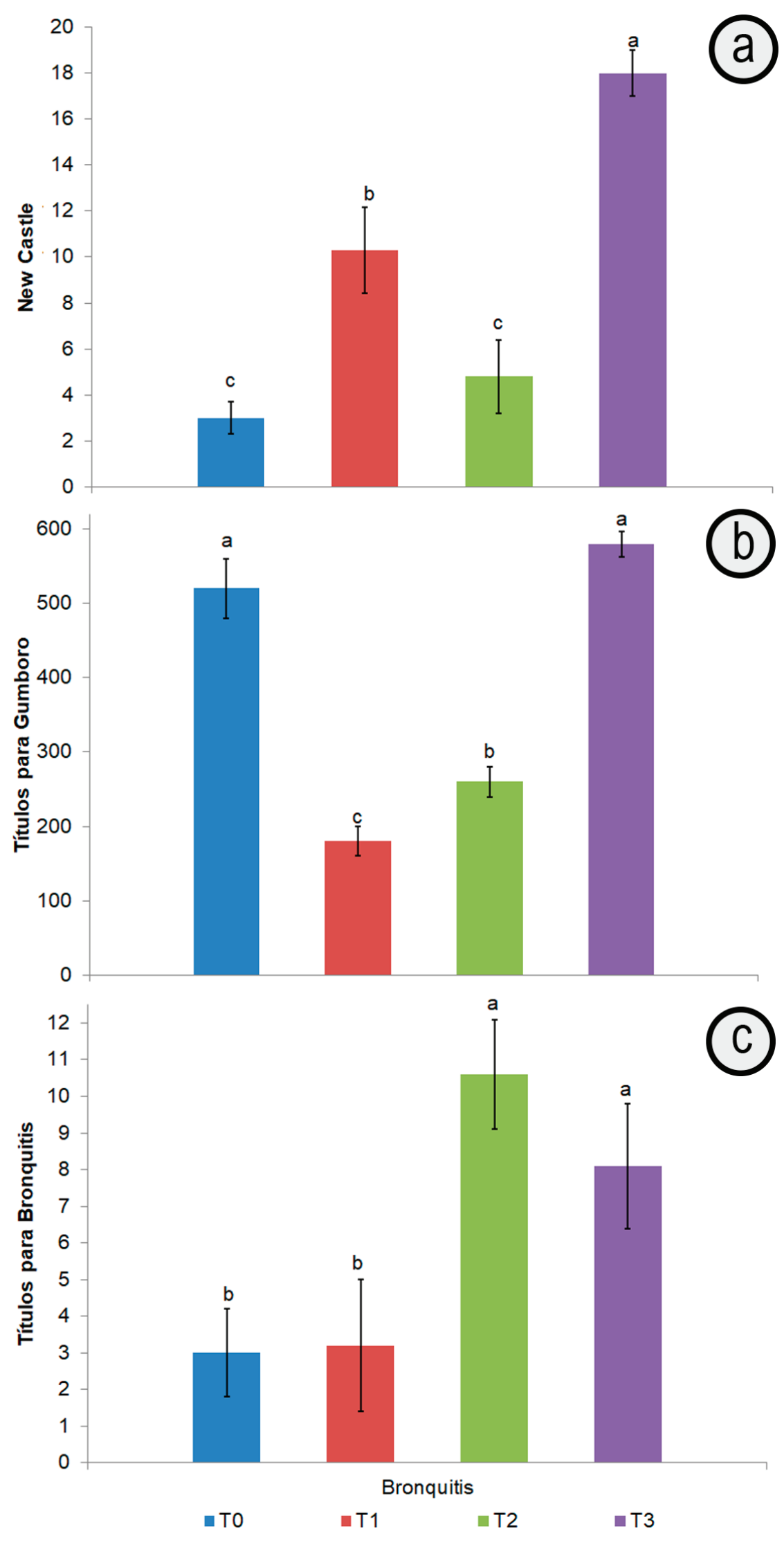

Figura 3. Determinación de títulos para las vacunas de: a. New castle; b. Gumboro y c. Bronquitis evaluados a los 35 días de vida. Letras distintas muestran diferencias estadísticas significativas $(\mathrm{p}<0,05)$. 


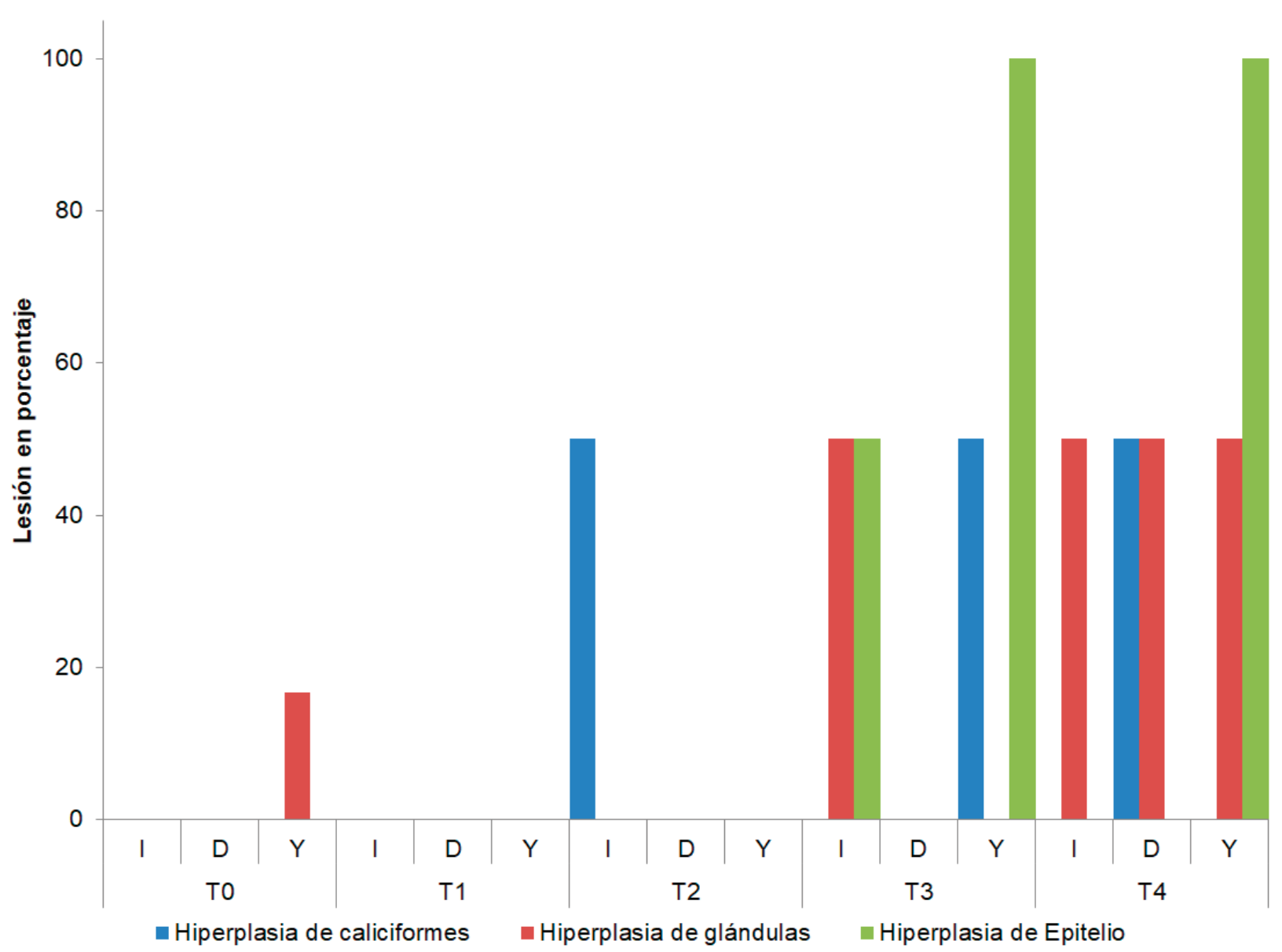

Figura 4. Resultados de las lesiones encontradas en el intestino delgado de las aves evaluadas. I: ileón, D: duodeno, Y: yeyuno.

\section{REFERENCIAS}

1. ABBAS, G.; IQBAL, M.A.; RIAZ, M.; SAJID, M.; ZAHID, O.; ABBAS, S.W.; RAZA, A.I. 2018. Comparative effect of different levels of probiotics (Protexin) on hematochemical profile in broilers. Advances in Zoology and Botany. 6(3):84-87.

https://doi.org/10.13189/azb.2018.060302

2. AL-KHALAIFAH, H. 2018. Benefits of probiotics and/or prebiotics for antibiotic-reduced poultry. Poultry Science. 97(11):3807-3815.

https://doi.org/10.3382/ps/pey160

3. BINGLE, C.; WILSON, K.; LUNN, H.; BARNES, F.A.; HIGH, A.S.; WALLACE, W.A.; BINGLE, L. 2010. Human LPLUNC1 is a secreted product of goblet cells and minor glands of the respiratory and upper aerodigestive tracts. Histochemistry and cell biology. 133(5):505-515. https://doi.org/10.1007/s00418-010-0683-0
4. CAI, Y.; SUYANANDANA, P.; SAMAN, P.; BENNO, Y. 1999. Classification and characterization of lactic acid bacteria isolated from the intestines of common carp and freshwater prawns. The J. general and applied microbiology. 45(4):177-184.

https://doi.org/10.2323/jgam.45.177

5. CASTRO-GONZÁLEZ, J.M.; CASTRO, P.; SANDOVAL, H.; CASTRO-SANDOVAL, D. 2019. Probiotic lactobacilli precautions. Frontiers Microbiology. 10:375. https://doi.org/10.3389/fmicb.2019.00375

6. CHAMPAGNE, C.P.; MOINEAU, S.; LAFLEUR, S.; SAVARD, T. 2017. The effect of bacteriophages on the acidification of a vegetable juice medium by microencapsulated Lactobacillus plantarum. Food microbiology. 63:28-34. https://doi.org/10.1016/j.fm.2016.10.036

7. COPPOLA, M.D.; GIL-TURNES, C. 2004. Probióticos e resposta imune. Ciência Rural. 34(4):1297-1303. https://doi.org/10.1590/S0103-84782004000400056 
8. DAHL, T.A.; MIDDEN, W.R.; HARTMAN, P.E. 1989. Comparison of killing of gram-negative and gram-positive bacteria by pure singlet oxygen. J. Bacteriology. 171(4):21882194.

https://doi.org/10.1128/jb.171.4.2188-2194.1989

9. ECKERT, C.; SERPA, V.G.; DOS-SANTOS, A.C.; DA-COSTA, S.M.; DALPUBEL, V.; LEHN, D.N.; DE-SOUZA, C.F. 2017. Microencapsulation of Lactobacillus plantarum ATCC 8014 through spray drying and using dairy whey as wall materials. LWT-food science and technology. 82:176-183. https://doi.org/10.1016/j.lwt.2017.04.045

10. GHASEMIAN, A.; ESLAMI, M.; SHAFIEI, M.; NAJAFIPOUR, S.; RAJABI, A. 2018. Probiotics and their increasing importance in human health and infection control. Reviews in Medical Microbiology. 29(4):153-158. https://doi.org/10.1097/MRM.0000000000000147

11. GÓMEZ-MASCARAQUE, L.G.; AMBROSIO-MARTÍN, J.; PEREZ-MASIÁ, R.; LÓPEZ-RUBIO, A. 2017. Impact of acetic acid on the survival of $L$. plantarum upon microencapsulation by coaxial electrospraying. J. Healthcare Engineering. 67(4):1234-1240. https://doi.org/10.1155/2017/4698079

12. GUNNARSSON, S.; MIE, A. 2018. Organic animal production-a tool for reducing antibiotic resistance? In Professionals in food chains (p.13). Wageningen Academic Publishers. https://doi.org/10.3920/978-90-8686-869-8

13. HAMID, M.A.; RAHMAN, M.A.; AHMED, S.; HOSSAIN, K.M. 2017. Status of poultry industry in Bangladesh and the role of private sector for its development. Asian J. Poultry Science. 11(1):1-13. https://doi.org/10.3923/ajpsaj.2017.1.13

14. INSTITUTO DE HIDROLOGÍA, METEREOLOGÍA Y ESTUDIOS AMBIENTALES, IDEAM. 2019. Consulta y Descarga de datos hidrometereológicos. Disponible desde Internet en: http://dhime.ideam.gov.co/atencionciudadano/ (con acceso el 10/07/2019).

15. JONES, P.J.; NIEMI, J.; CHRISTENSEN, J.P.; TRANTER, R.B.; BENNETT, R.M. 2019. A review of the financial impact of production diseases in poultry production systems. Animal Production Science. 59(9):1585-1597. https://doi.org/10.1071/AN18281

16. JURADO-GÁMEZ, H.; CALPA, F.; CHASPUENGAL, A. 2014. Determinación in vitro de la acción probiótica de Lactobacillus plantarum sobre Yersinia pseudotuberculosis aislada de Cavia porcellus. Rev. Facultad de Medicina Veterinaria y de Zootecnia. 61(3):34-45.

http://dx.doi.org/10.15446/rfmvz.v61n3.46872

17. MATOS, R.C.; SCHWARZER, M.; GERVAIS, H.; COURTIN, P.; JONCOUR, P.; GILLET, B.; CHAPOT-CHARTIER, M.P. 2017. D-Alanylation of teichoic acids contributes to Lactobacillus plantarum-mediated Drosophila growth during chronic undernutrition. Nature Microbiology. 2(12):16351647.

https://doi.org/10.1038/s41564-017-0038-x

18. MILIAN, G. 2008. Empleo de probióticos a base de Bacillus sp y sus endosporas en la producción avícola. Revista Cubana de Ciencias Agrícolas. 42(2):1-16.

19. MUHAMMAD, Z.; RAMZAN, R.; HUO, G.C.; TIAN, H.; BIAN, X. 2017. Integration of polysaccharidethermoprotectant formulations for microencapsulation of Lactobacillus plantarum, appraisal of survivability and physico-biochemical properties during storage of spray dried powders. Food hydrocolloids. 66:286-295. https://doi.org/10.1016/j.foodhyd.2016.11.040

20. OVERBECK, T.J.; WELKER, D.L.; HUGHES, J.E.; STEELE, J.L.; BROADBENT, J.R. 2017. Transient MutS-Based hypermutation system for adaptive evolution of Lactobacillus casei to low pH. Appl. Environ. Microbiol. 83(20):e01120-17. https://doi.org/10.1128/AEM.01120-17

21. PALACHUM, W.; CHOORIT, W.; CHISTI, Y. 2018. Accumulation of conjugated linoleic acid in Lactobacillus plantarum WU-P19 is enhanced by induction with linoleic acid and chitosan treatment. Annals of microbiology. 68(10):611-624. https://doi.org/10.1007/s13213-018-1368-5

22. PARRA-HUERTAS, R.A. 2010. Bacterias ácido lácticas: papel funcional en los alimentos. Facultad de Ciencias Agropecuarias. 8(1):67-78.

23. RODRÍGUEZ-BARONA, S.; GIRALDO, G.I.; MONTES, L.M. 2016. Encapsulación de alimentos probióticos mediante liofilización en presencia de prebióticos. Información Tecnológica. 27(6):135-144. http://dx.doi.org/10.4067/S0718-07642016000600014

24. RODRÍGUEZ, P.A.; ARENAS, R. 2018. Hans Christian Gram y su tinción. Dermatología Cosmética, Médica y Quirúrgica. 2:166-167.

25. SOBCZAK, J.; STANGIERSKI, J.; MAREK, P.; KIJOWSKI, J. 2018. Effect of probiotic administration on productivity and quality of broiler chicken meat. Animal Nutrition and 
Feed Technology. 18(1):79-88.

https://doi.org/10.5958/0974-181X.2018.00007.0

26. STEFANIAK, T.; MADEJ, J.P.; GRACZYK, S.; SIWEK, M.; LUKASZEWICZ, E.; KOWALCZYK, A.; BEDNARCZYK, M. 2019. Selected prebiotics and synbiotics administered in ovo can modify innate immunity in chicken broilers. BMC Veterinary Research. 15(1):105116.

https://doi.org/10.1186/s12917-019-1850-8

27. WANG, G.; HUANG, W.; XIA, Y.; XIONG, Z.; AI, L. 2019. Cholesterol-lowering potentials of Lactobacillus strain overexpression of bile salt hydrolase on high cholesterol diet-induced hypercholesterolemic mice. Food \& Function. 10(3):1684-1695.

https://doi.org/10.1039/C8FO02181c
28. WANG, W.; CHEN, J.; ZHOU, H.; WANG, L.; DING, S.; WANG, Y.; LI, A. 2018. Effects of microencapsulated Lactobacillus plantarum and fructooligosaccharide on growth performance, blood immune parameters, and intestinal morphology in weaned piglets. Food and agricultural immunology. 29(1):84-94. https://doi.org/10.1080/09540105.2017.1360254

29. ZHANG, L.; ZHANG, L.; ZENG, X.; ZHOU, L.; CAO, G.; YANG, C. 2016. Effects of dietary supplementation of probiotic, Clostridium butyricum, on growth performance, immune response, intestinal barrier function, and digestive enzyme activity in broiler chickens challenged with Escherichia coli K88. J. Animal Science and Biotechnology. 7(1):3-15.

https://doi.org/10.1186/s40104-016-0061-4 